\title{
LEV BIRBRAIR
}

\section{MARINA SOBOLEVSKY}

\section{Realization of Hölder complexes}

Annales de la faculté des sciences de Toulouse $6^{e}$ série, tome $8, \mathrm{n}^{\circ} 1$ (1999), p. 35-44

<http://www.numdam.org/item?id=AFST_1999_6_8_1_35_0>

(C) Université Paul Sabatier, 1999, tous droits réservés.

L'accès aux archives de la revue «Annales de la faculté des sciences de Toulouse » (http://picard.ups-tlse.fr/ annales/) implique l'accord avec les conditions générales d'utilisation (http://www.numdam.org/conditions). Toute utilisation commerciale ou impression systématique est constitutive d'une infraction pénale. Toute copie ou impression de ce fichier doit contenir la présente mention de copyright.

\section{NumDam}

Article numérisé dans le cadre du programme

Numérisation de documents anciens mathématiques

http://www.numdam.org/ 


\title{
Realization of Hölder Complexes ${ }^{(*)}$
}

\author{
LEV Birbrair and Marina SoboleVSKY $(1)$
}

\begin{abstract}
RÉsuMÉ. - Un complexe de Hölder est un graphe fini tel qu'à chaque arête est associé un nombre rationnel positif et on sait que c'est un invariant bi-lipschitzien des ensembles semi-algébriques singuliers de dimension 2. On montre dans cet article que tout complexe de Hölder peut être réalisé comme un ensemble semi-algébrique de dimension 2. Pour ce faire on plonge le graphe dans un tore de dimension $n$ qu'on fait contracter sur un point singulier de telle sorte que les générateurs s'évanouissent avec les vitesses rationnelles et différentes.
\end{abstract}

Abstract. - Hölder Complex, a graph and a rationaly-valued function on the set of the edges of the graph, is a bi-Lipschitz invariant of 2dimensional semialgebraic singular sets. Here we prove that each Hölder Complex can be realized as a 2-dimensional semialgebraic set. For this purpose we embed the graph to an $n$-dimensional torus. The torus is vanishing in a singular point such that the generators are vanishing with different rational rates.

\section{Introduction}

The paper is devoted to the local geometry of 2-dimensional semialgebraic sets. The local bi-Lipschitz classification theorem is proved in [1]. The main notion of the classification is a so-called Geometric Hölder Complex. It is a local version of a simplicial complex with some additional geometric information (see the definition below). A Hölder Complex can be considered as a combinatorial object - a finite graph with a rational-valued function defined on the set of edges.

(*) Reçu le 7 avril 1997 , accepté le 30 septembre 1997

(1) Departamento de Matematica, Universidade Federal do Ceara, CEP 60455-760 BR-Fortaleza CE (Brazil)

e-mail: lev@mat.ufc.br

e-mail: marina@mat.ufc.br 


\section{Birbrair and M. Sobolevsky}

The following question is natural. Let us define a Hölder Complex in a combinatorial way. Does it correspond to some semialgebraic set?

The answer is positive. To prove the Realization theorem we define a semialgebraic set $T\left(\beta_{1}, \ldots, \beta_{k}\right)$. It is a generalization of the real algebraic set which gives an example of the noncoincidence of $L_{p}$-cohomology and Intersection Homology [2]. The set $T\left(\beta_{1}, \ldots, \beta_{k}\right)$ has a toric link at the singular point and all generators of the torus have different vanishing rates in this point. It gives us a possibility to separate vanishing rates of all edges of a Hölder Complex.

\section{Definitions and notations}

Let us recall some definitions from [1]. Let $\Gamma$ be a connected graph without loops, $V_{\Gamma}=\left\{a_{1}, a_{2}, \ldots, a_{k}\right\}$ be the set of vertices and $E_{\Gamma}=$ $\left\{g_{1}, g_{2}, \ldots, g_{r}\right\}$ be the set of edges of the graph.

Definition 2.1. - A Hölder Complex $(\Gamma, \beta)$ is a graph $\Gamma$ with an associated function $\beta: E_{\Gamma} \rightarrow[1, \infty[\cap Q$ (here $Q$ is the ring of rational numbers).

Definition 2.2.- A Curvilinear triangle $T$ is a subset of $\mathbb{R}^{n}$ homeomorphic to a 2-dimensional simplex satisfying the following properties.

1) Each internal (in the induced topology) point $t \in T$ has an open neighbourhood $U_{t} \subset T$ such that $U_{t}$ is a smooth 2-dimensional submanifold of $\mathbb{R}^{n}$ at each point $t^{\prime} \in U_{t}$.

2) The boundary of $T$ is a union of three analytic curves $\gamma_{1}, \gamma_{2}, \gamma_{3}$ such that $\gamma_{i}$ (for $i=1,2,3$ ) has a neighbourhood at each internal (in the induced from $\mathbb{R}$ topology on $\gamma_{i}$ ) point which is a smooth 1-dimensional submanifold of $\mathbb{R}^{n}$.

3) Locally $T$ is a smooth manifold with a boundary at each smooth point of the boundary.

Boundary points of $\gamma_{i}$ we call vertices of $T$.

Definition 2.3. - A standard $\beta$-Hölder triangle $\mathrm{ST}_{\beta}$ is a subset of the plane $\mathbb{R}^{2}$ bounded by the following curves:

$$
\begin{gathered}
\{y=0\}, \quad\left\{y=x^{\beta}\right\}, \quad\{x=1\} . \\
-36-
\end{gathered}
$$




\section{Realization of Hölder Complexes}

Let us consider a cone $C \Gamma$ over $\Gamma$. Let $A_{0}$ be the vertex of $C \Gamma$. We can consider $C \Gamma$ as a topological space with the standard topology of a simplicial complex.

Definition 2.4. - A subset $H(\Gamma, \beta) \subset \mathbb{R}^{n}$ is called a Geometric Hölder Complex corresponding to $(\Gamma, \beta)$ if it satisfies the following conditions.

1) $H(\Gamma, \beta)$ is a subanalytic subset of $\mathbb{R}^{n}$.

2) There exists a homeomorphism $F: C \Gamma \rightarrow H(\Gamma, \beta)$.

3) The set $H(\Gamma, \beta) \cap S_{F\left(A_{0}\right), r}$ is empty or homeomorphic to $\Gamma$, for every $r$. (We use the notation $S_{F\left(A_{0}\right), r}$ for the sphere centered at the point $F\left(A_{0}\right)$ with the radius $r$.)

4) The image of the triangle $\left(A_{0}, a_{i}, a_{j}, g\right)$ (where $a_{i}$ and $a_{j}$ are vertices of $\Gamma, g$ is the edge connecting $a_{i}$ and $a_{j},\left(A_{0}, a_{i}, a_{j}, g\right)$ is the subcone of $C \Gamma$ over $g$ ) has the following properties :

(a) $F\left(A_{0}, a_{i}, a_{j}, g\right)$ is a subanalytic subset of $\mathbb{R}^{n}$;

(b) $F\left(A_{0}, a_{i}, a_{j}, g\right)$ is subanalytically bi-Lipschitz equivalent to the standard $\beta(g)$-Hölder triangle $\mathrm{ST}_{\beta(g)}$;

(c) let $L: \mathrm{ST}_{\beta(g)} \rightarrow F\left(A_{0}, a_{i}, a_{j}, g\right)$ be this subanalytic bi-Lipschitz map; then

$$
L(0,0)=F\left(A_{0}\right), \quad L(1,0)=F\left(a_{i}\right), \quad L(1,1)=F\left(a_{j}\right) .
$$

Definition 2.5. - A $\beta$-Hölder triangle $\mathrm{HT}_{\beta}$ is a subset of $\mathbb{R}^{n}$ satisfying the following conditions.

1) $\mathrm{HT}_{\beta}$ is a curvilinear triangle.

2) $\mathrm{HT}_{\beta}$ is bi-Lipschitz equivalent to some standard $\beta$-Hölder triangle $\mathrm{ST}_{\beta}$.

3) The bi-Lipschitz map $L: \mathrm{ST}_{\beta} \rightarrow \mathrm{HT}_{\beta}$ is subanalytic. (The image of the point $(0,0)$ is called a Hölder vertex of $\mathrm{HT}_{\beta}$.)

Definition 2.6. - A standard $\beta$-horn $\mathrm{SH}_{\beta}$ (here $\beta \in \mathbb{Q} \cap[1,+\infty[)$ is a semialgebraic set in $\mathbb{R}^{3}$ defined by the following conditions:

$$
\left(x_{1}^{2}+x_{2}^{2}\right)^{q}=y^{2 p}, \quad 0 \leq y \leq 1,
$$

$\left(x_{1}, x_{2}, y\right)$ are coordinates of a point in $\mathbb{R}^{3}$ and $\beta=p / q$ with $\operatorname{GCD}(p, q)=1$. 
We proved in [1] that every 2-dimensional semialgebraic (as well as semianalytic and subanalytic) set $X$ is a Geometric Hölder Complex in a neighbourhood of a given point $a_{0} \in X$ corresponding to some Hölder Complex. Here we are going to prove the following result.

Realization theorem. - Let $(\Gamma, \beta)$ be a Hölder Complex. Then there exist a semialgebraic 2-dimensional set $X \subset \mathbb{R}^{n}$, a point $a_{0} \in X$ and $\varepsilon>0$ such that $X \cap B_{a_{0}, \varepsilon}$ is a Geometric Hölder Complex corresponding to the Hölder Complex $(\Gamma, \beta)$ (here $B_{a_{0}, \varepsilon}$ is a closed ball in $\mathbb{R}^{n}$ centered at the point $a_{0}$ with the radius $\varepsilon$ ).

\section{The set $T\left(\beta_{1}, \ldots, \beta_{k}\right)$. Polar maps}

We consider the space $\mathbb{R}^{2 k+1}$ with coordinates $\left(x_{1}, y_{1}, x_{2}, y_{2}, \ldots\right.$, $\left.x_{k}, y_{k}, z\right)$. Let $D\left(\beta_{1}, \ldots, \beta_{k}\right)$ (here $\beta_{i}=p_{i} / q_{i}$ with $p_{i}, q_{i} \in \mathbb{Z}$ and $\operatorname{GCD}\left(p_{i}, q_{i}\right)=1$ ) be a subvariety of $\mathbb{R}^{2 k+1}$ given by the following equations:

$$
\begin{gathered}
z^{2 p_{1}}=\left(x_{1}^{2}+y_{1}^{2}\right)^{q_{1}} \\
\vdots \\
z^{2 p_{i}}=\left(x_{i}^{2}+y_{i}^{2}\right)^{q_{i}} \\
\vdots \\
z^{2 p_{k}}=\left(x_{k}^{2}+y_{k}^{2}\right)^{q_{k}} .
\end{gathered}
$$

(The set described in the paper [2] is a special 3-dimensional example of $D\left(\beta_{1}, \beta_{2}\right)$.)

Let

$$
T\left(\beta_{1}, \ldots, \beta_{k}\right)=D\left(\beta_{1}, \ldots, \beta_{k}\right) \cap\{z \geq 0\}
$$

LEMMA 3.1

1) $\operatorname{dim} T\left(\beta_{1}, \ldots, \beta_{k}\right)=k+1$.

2) The link of $T\left(\beta_{1}, \ldots, \beta_{k}\right)$ at the point $(0, \ldots, 0)$ is homeomorphic to $T^{k}$ (a k-dimensional torus).

(Remind that the link of $T\left(\beta_{1}, \ldots, \beta_{k}\right)$ is the intersection of $T\left(\beta_{1}, \ldots, \beta_{k}\right)$ with a small sphere centered at $(0, \ldots, 0)$.) 


\section{Realization of Hölder Complexes}

\section{Proof}

1) Consider a section of $T\left(\beta_{1}, \ldots, \beta_{k}\right)$ by the plane $z=c$. We obtain the equations

$$
x_{i}^{2}+y_{i}^{2}=c_{i}
$$

where $c_{i}=c^{2 p_{i} / q_{i}}$. Clearly, these equations define a $k$-dimensional torus. The variety $T\left(\beta_{1}, \ldots, \beta_{k}\right)$ we obtain as a suspension of it. So, (1) is proved.

2) Let $r(z)$ be a function defined in the following way:

$$
r(z)=\sqrt{z^{2}+\sum_{i=1}^{k} z^{\beta_{i}}} .
$$

This function $r(z)$ is a one-to-one function, for small $z$. Thus, for sufficiently small $\varepsilon>0$, the link $T\left(\beta_{1}, \ldots, \beta_{k}\right) \cap S_{0, \varepsilon}$ is equal to the torus $T\left(\beta_{1}, \ldots, \beta_{k}\right) \cap\left\{\left(x_{1}, y_{1}, \ldots, x_{k}, y_{k}, z\right) \in \mathbb{R}^{2 k+1} \mid z=r^{-1}(\varepsilon)\right\}$.

Each point of $T\left(\beta_{1}, \ldots, \beta_{k}\right)$ has uniquely defined polar coordinates $\left(\psi_{1}, \psi_{2}, \ldots, \psi_{k}, z\right): \psi_{i}$ is the angle coordinate of the corresponding point of the circle $x_{i}^{2}+y_{i}^{2}=c_{i}$ and $z$ is a $z$-coordinate in $\mathbb{R}^{2 k+1}$. Let $x^{0}=$ $\left(\psi^{0}, z^{0}\right)=\left(\psi_{1}^{0}, \ldots, \psi_{k}^{0}, z^{0}\right)$ be a point of $T\left(\beta_{1}, \ldots, \beta_{k}\right)$. Let $L_{x^{0}}$ be a curve on $T\left(\beta_{1}, \ldots, \beta_{k}\right)$ defined as follows:

$$
L_{x^{0}}=\left\{\left(\psi_{1}, \psi_{2}, \ldots, \psi_{k}, z\right) \mid \psi_{1}=\psi_{1}^{0}, \ldots, \psi_{k}=\psi_{k}^{0}\right\}
$$

We call $L_{x^{0}}$ a polar line generated by $x^{0}$. Now we can define a polar map in the following way.

Denote, for $\varepsilon>0$, the set

$$
T\left(\beta_{1}, \ldots, \beta_{k}\right) \cap\left\{\left(x_{1}, y_{1}, \ldots, x_{k}, y_{k}, z\right) \in \mathbb{R}^{2 k+1} \mid z \leq \varepsilon\right\}
$$

by $T^{\varepsilon}\left(\beta_{1}, \ldots, \beta_{k}\right)$. Let $P_{\varepsilon_{1}, \varepsilon_{2}}: T^{\varepsilon_{1}}\left(\beta_{1}, \ldots, \beta_{k}\right) \rightarrow T^{\varepsilon_{2}}\left(\beta_{1}, \ldots, \beta_{k}\right)$ be a map defined as follows:

$$
P_{\varepsilon_{1}, \varepsilon_{2}}\left(\psi_{1}, \ldots, \psi_{k}, z\right)=\left(\psi_{1}, \ldots, \psi_{k}, \frac{\varepsilon_{1}}{\varepsilon_{2}} z\right)
$$

We call $P_{\varepsilon_{1}, \varepsilon_{2}}$ a polar map. Observe that $P_{\varepsilon_{1}, \varepsilon_{2}}$ is a bi-Lipschitz map. 
Remark 3.1. $-T\left(\beta_{1}\right)$ is an usual $\beta_{1}$-horn.

Remark 3.2. $-T\left(\beta_{1}, \ldots, \beta_{k}\right)$ is included to $T\left(\beta_{1}, \ldots, \beta_{k}, \ldots, \beta_{n}\right)$ (here $n \geq k+1$ ) as a semialgebraic subset defined by the following equations $\psi_{k+1}=b_{1}, \psi_{k+2}=b_{2}, \ldots, \psi_{n}=b_{n-k}, b_{1}, \ldots, b_{n-k} \in \mathbb{R}$.

\section{Proof of the Realization theorem}

We use the induction on the number of edges. Suppose that each Hölder Complex $(\Gamma, \beta)$ whose graph $\Gamma$ has less or equal than $k$ edges is realized as a semialgebraic subset of $T\left(\beta_{1}, \ldots, \beta_{k}\right)$ such that all vertices of $\Gamma$ belong to the section by the plane $z=1$ and, for each vertex $a$, we have $\psi_{i}(a)=0$ or $\psi_{i}(a)=\pi$. (We can identify the graph $\Gamma$ and its image by the map $F$; see Definition 2.4.)

For $k=1$, the assertion is trivial: $\Gamma$ has two vertices $a_{1}$ and $a_{2}$. Set $\psi\left(a_{1}\right)=0, \psi\left(a_{2}\right)=\pi$ and the edge connecting $a_{1}$ and $a_{2}$ be a half-circle. So, $(\Gamma, \beta)$ is realized as a half of the standard $\beta$-horn.

Now consider a Hölder Complex $(\Gamma, \beta)$ such that $\Gamma$ has $(k+1)$ edges. Let $g$ be an edge such that $\beta(g)=\min _{\tilde{g} \in E_{\Gamma}} \beta(\tilde{g})$. Let us consider a graph $\widetilde{\Gamma}=\Gamma-g$. We have two possibilities: $\widetilde{\Gamma}$ is a connected graph or $\widetilde{\Gamma}$ is not connected.

Suppose that $\widetilde{\Gamma}$ is not connected. Then it is a union of two connected components $\widetilde{\Gamma}=\widetilde{\Gamma}^{1} \cup \widetilde{\Gamma}^{2}$ (we include also a case when one of these components is just a vertex). We can suppose that $g_{1}, \ldots, g_{\ell} \in E_{\tilde{\Gamma}^{1}}$, $g_{\ell+1}, \ldots, g_{k} \in E_{\tilde{\Gamma}_{2}}, g_{k+1}=g$. Now consider a set $T\left(\beta_{1}, \ldots, \beta_{k}, \beta(g)\right)$ and a section of that by the plane $z=1$. This section is a $(k+1)$ dimensional torus (see the proof of the Lemma 3.1). By the induction hypotheses, the subcomplex $\left(\widetilde{\Gamma}^{1}, \widetilde{\beta}^{1}\right)$, where $\widetilde{\beta}^{1}=\left.\beta\right|_{\tilde{\Gamma}^{1}}$, can be realized as a semialgebraic subset of $T\left(\beta_{1}, \ldots, \beta_{k}\right)$ which can be considered as a semialgebraic subset of $T\left(\beta_{1}, \ldots, \beta_{k}, \beta(g)\right)$ given by the equation $\psi_{k+1}=0$ (see the Remark 3.2). By the same way, $\left(\widetilde{\Gamma}^{2}, \widetilde{\beta}^{2}\right)$, where $\widetilde{\beta}^{2}=\left.\beta\right|_{\tilde{\Gamma}^{2}}$, can be realized as a semialgebraic subset of $T\left(\beta_{1}, \ldots, \beta_{k}\right)$ which can be considered as a semialgebraic subset of $T\left(\beta_{1}, \ldots, \beta_{k}, \beta(g)\right)$ given by the equation $\psi_{k+1}=\pi$. Suppose that $g$ connects vertices $a_{1} \in \widetilde{\Gamma}^{1}$ and $a_{2} \in \widetilde{\Gamma}^{2}$; 
let $a_{1}$ has polar coordinates $\left(\psi_{1}\left(a_{1}\right), \ldots, \psi_{k}\left(a_{1}\right), 0\right)$ and let $a_{2}$ has polar coordinates $\left(\psi_{1}\left(a_{2}\right), \ldots, \psi_{k}\left(a_{2}\right), \pi\right)$. We connect these two vertices by the following curve $\Psi(\theta)=\left\{\psi_{1}(\theta), \psi_{2}(\theta), \ldots, \psi_{k+1}(\theta), 1\right\}$ where

$$
\psi_{k+1}(\theta)=\theta, \quad \psi_{i}(\theta)= \begin{cases}\psi_{i}\left(a_{1}\right) & \text { if } \psi_{i}\left(a_{1}\right)=\psi_{i}\left(a_{2}\right) \\ \theta & \text { if } \psi_{i}\left(a_{1}\right)=0 \text { and } \psi_{i}\left(a_{2}\right)=\pi \\ \pi+\theta & \text { if } \psi_{i}\left(a_{1}\right)=\pi \text { and } \psi_{i}\left(a_{2}\right)=0\end{cases}
$$

$1 \leq i \leq k, \theta \in[0, \pi]$. Clearly, $\Psi(0)=a_{1}$ and $\Psi(\pi)=a_{2}$. Define

$$
H_{\beta(g)}:=\bigcup_{\theta} L_{\Psi(\theta)},
$$

the union of the polar lines generated by $\Psi(\theta)$.

Lemma 4.1. - The set $H_{\beta(g)}$ is a $\beta(g)$-Hölder triangle.

Proof. - $H_{\beta(g)}$ is a semialgebraic set because it is defined by the system (3) which can be written as a system of algebraic equations and inequalities in terms of variables $x_{i}, y_{i}$, for $1 \leq i \leq k+1$, and by the inequalities $0 \leq z \leq 1$. Hence, $H_{\beta(g)} \cap B_{0, \varepsilon}$ (here $B_{0, \varepsilon}$ is a closed ball in $\mathbb{R}^{2 k+3}$ centered at 0 with the radius $\varepsilon$ ) is a Geometric Hölder Complex $H(\bar{\Gamma}, \alpha)$ corresponding to some graph $\bar{\Gamma}$ with some rational-valued function $\alpha$ defined on its edges [1]. Since $H_{\beta(g)}$ is a curvilinear triangle (by the construction), $H_{\beta(g)} \cap B_{0, \varepsilon_{0}}$, for sufficiently small $\varepsilon_{0} \leq \varepsilon$, is bi-Lipschitz equivalent to the standard $\alpha_{0}$-Hölder triangle where $\alpha_{0}=\min _{\bar{g} \in E_{\bar{\Gamma}}} \alpha(\bar{g})$ [1, Second Structural Lemma]. But $H_{\beta(g)} \cap B_{0, \varepsilon_{0}}$ is bi-Lipschitz equivalent to $H_{\beta(g)}$ (the biLipschitz equivalence is given by the polar map $P_{\varepsilon_{0}, 1}$ ).

To complete the proof of the lemma we must show that $\alpha_{0}=\beta(g)$. Let $\gamma_{\varepsilon}$ be the equidistant line in $H_{\beta(g)}$, namely $\gamma_{\varepsilon}=H_{\beta(g)} \cap S_{0, \varepsilon}$. By [1], there exists a subanalytic bi-Lipschitz map $\Upsilon: H_{\beta(g)} \rightarrow \mathrm{ST}_{\alpha_{0}}$ such that $\Upsilon\left(\gamma_{\varepsilon}\right)=\mathrm{ST}_{\alpha_{0}} \cap\left\{(x, y) \in \mathbb{R}^{2} \mid x=\varepsilon\right\}$. Denote by $\ell\left(\gamma_{\varepsilon}\right)$ the length of $\gamma_{\varepsilon}$. Since $\Upsilon$ is a bi-Lipschitz map, we have

$$
c_{1} \varepsilon^{\alpha_{0}} \leq \ell\left(\gamma_{\varepsilon}\right) \leq c_{2} \varepsilon^{\alpha_{0}},
$$

for some positive constants $c_{1}$ and $c_{2}$. To prove that $\alpha_{0}=\beta(g)$ we will compute the length of $\gamma_{\varepsilon}$ from another side. Consider the function

$$
r(z)=\sqrt{z^{2}+\sum_{i=1}^{k+1} z^{p_{i} / q_{i}}}
$$




\section{Birbrair and M. Sobolevsky}

which is a one-to-one function, for small $z$. So, $r^{-1}(\varepsilon)$ is a well-defined function, for small $\varepsilon$. By the Lemma 3.1,

$$
\gamma_{\varepsilon}=H_{\beta(g)} \cap\left\{\left(x_{1}, y_{1}, \ldots, x_{k+1}, y_{k+1}, z\right) \in \mathbb{R}^{2 k+3} \mid z=r^{-1}(\varepsilon)\right\} .
$$

Consider the following set

$$
\begin{aligned}
T^{\varepsilon} & =T\left(\beta_{1}, \ldots, \beta_{k}, \beta(g)\right) \\
& \cap\left\{\left(x_{1}, y_{1}, \ldots, x_{k+1}, y_{k+1}, z\right) \in \mathbb{R}^{2 k+1} \mid z=r^{-1}(\varepsilon)\right\} .
\end{aligned}
$$

It is a smooth manifold homeomorphic to a $(k+1)$-dimensional torus. The equidistant line $\gamma_{\varepsilon}$ belongs to this set. There are $(k+1)$ differencial 1-forms $\mathrm{d} \psi_{1}^{\varepsilon}, \ldots, \mathrm{d} \psi_{k}^{\varepsilon}$ and $\mathrm{d} \psi_{k+1}^{\varepsilon}$ on $T^{\varepsilon}$ corresponding to the coordinate system $\left\{\psi_{1}, \ldots, \psi_{k}, \psi_{k+1}\right\}$. By $(3)$, we have

$$
\begin{gathered}
\ell\left(\gamma_{\varepsilon}\right)=\int_{\gamma_{\varepsilon}} \sum_{i=1}^{k+1} m_{i} \mathrm{~d} \psi_{i}^{\varepsilon} \quad \text { where } m_{i}= \begin{cases}1 & \text { if } \psi_{i}\left(a_{1}\right) \neq \psi_{i}\left(a_{2}\right) \\
0 & \text { if } \psi_{i}\left(a_{1}\right)=\psi_{i}\left(a_{2}\right),\end{cases} \\
\int_{\gamma_{\varepsilon}} \sum_{i=1}^{k+1} m_{i} \mathrm{~d} \psi_{i}^{\varepsilon} \leq \sum_{i=1}^{k+1} \int_{\gamma_{\varepsilon}} m_{i} \mathrm{~d} \psi_{i}^{\varepsilon} .
\end{gathered}
$$

By the definition of the equidistant line $\gamma_{\varepsilon}$,

$$
\int_{\gamma_{\varepsilon}} m_{i} \mathrm{~d} \psi_{i}^{\varepsilon}=m_{i} \pi z^{\beta_{i}}
$$

Using the above formula we obtain

$$
\ell\left(\gamma_{\varepsilon}\right) \leq \sum_{i=1}^{k+1} m_{i} \pi z^{\beta_{i}} .
$$

If $z$ sufficiently small $(z<1)$ there exists $\widetilde{C}_{2}>0$ such that

$$
\ell\left(\gamma_{\varepsilon}\right) \leq \sum_{i=1}^{k+1} m_{i} \pi z^{\beta_{i}} \leq \widetilde{C}_{2} z^{\beta(g)},
$$

because $\beta(g)=\min _{1 \leq i \leq k+1} \beta_{i}$.

By the definition of the function $r(\varepsilon)$, we have $r(\varepsilon)=a \varepsilon+o(\varepsilon)$, with $a>0$. 


\section{Realization of Hölder Complexes}

Hence, $\ell\left(\gamma_{\varepsilon}\right) \leq C_{2}^{\prime} \varepsilon^{\beta(g)}$, where $C_{2}^{\prime}=a \widetilde{C}_{2}$. To obtain an estimate of $\ell\left(\gamma_{\varepsilon}\right)$ from below let us go back to the formulas (3)

$$
\ell\left(\gamma_{\varepsilon}\right)=\int_{\gamma_{\varepsilon}} \sum_{i=1}^{k+1} m_{i} \mathrm{~d} \psi_{i}^{\varepsilon} \geq \int_{\gamma_{\varepsilon}} m_{k+1} \mathrm{~d} \psi_{k+1}^{\varepsilon} .
$$

By (3), $m_{k+1}=1$. Thus,

$$
\ell\left(\gamma_{\varepsilon}\right) \geq \int_{\gamma_{\varepsilon}} \mathrm{d} \psi_{k+1}^{\varepsilon}=\pi z^{\beta(g)} \geq C_{1}^{\prime} \varepsilon^{\beta(g)}
$$

for some positive constant $C_{1}^{\prime}$. So,

$$
C_{1}^{\prime} \varepsilon^{\beta(g)} \leq \ell\left(\gamma_{\varepsilon}\right) \leq C_{2}^{\prime} \varepsilon^{\beta(g)} .
$$

From (4) and (5) we obtain that $\beta(g)=\alpha_{0}$.

Lemma 4.1 is proved.

- Thus, the realization of $(\Gamma, \beta)$ is given by the union of the realizations of $\left(\widetilde{\Gamma}^{1}, \widetilde{\beta}^{1}\right),\left(\widetilde{\Gamma}^{2}, \widetilde{\beta}^{2}\right)$ and $H_{\beta(g)}$. It is a semialgebraic set because it is a finite union of semialgebraic sets.

Now consider the second case: $\widetilde{\Gamma}$ is a connected graph. In this case, by the induction hypotheses, $(\widetilde{\Gamma}, \widetilde{\beta})$ (where $\widetilde{\beta}=\left.\beta\right|_{\tilde{\Gamma}}$ ) can be realized as a semialgebraic subset of $T\left(\beta_{1}, \ldots, \beta_{k}\right)$ which can be considered as a semialgebraic subset of $T\left(\beta_{1}, \ldots, \beta_{k}, \beta(g)\right)$ defined by the equation $\psi_{k+1}=0$. The edge $g$ connects two vertices $a_{1}$ and $a_{2}$. Now we can glue the realization of $(\widetilde{\Gamma}, \widetilde{\beta})$ and the curvilinear triangle $H_{\beta(g)}$ generated by the curve $\Psi(\theta)=\left\{\psi_{1}(\theta), \psi_{2}(\theta), \ldots, \psi_{k+1}(\theta)\right\}$ :

$$
\psi_{k+1}(\theta)=\theta \text { and } \psi_{i}(\theta)= \begin{cases}\psi_{i}\left(a_{1}\right) & \text { if } \psi_{i}\left(a_{1}\right)=\psi_{i}\left(a_{2}\right) \\ \frac{\theta}{2} & \text { if } \psi_{i}\left(a_{1}\right)=0 \text { and } \psi_{i}\left(a_{2}\right)=\pi \\ \pi+\frac{\theta}{2} & \text { if } \psi_{i}\left(a_{1}\right)=\pi \text { and } \psi_{i}\left(a_{2}\right)=0\end{cases}
$$

for $1 \leq i \leq k, \theta \in[0,2 \pi], a_{1}=\left(\psi_{1}\left(a_{1}\right), \ldots, \psi_{k}\left(a_{1}\right), 0\right)$ and $a_{2}=$ $\left.\left(\psi_{1}\left(a_{2}\right), \ldots, \psi_{k}\left(a_{2}\right), \pi\right)\right)$.

Set $H_{\beta(g)}:=\bigcup_{\theta} L_{\Psi(\theta)}$. By the same arguments as in the Lemma 4.1, we can prove that $H_{\beta(g)}$ is a $\beta(g)$-Hölder triangle. 
The union of the realization of $(\widetilde{\Gamma}, \widetilde{\beta})$ and $H_{\beta(g)}$ is a semialgebraic realization of $(\Gamma, \beta)$.

The Realization theorem is proved.

\section{Acknowledgments}

The authors were supported by CNPq grants N 300985/93-2(RN) and

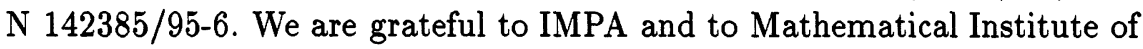
PUC-Rio, where this work was done.

\section{References}

[1] BIRBRAIR (L.) .- Local bi-Lipschitz classification of 2-dimensional semialgebraic sets, Preprint I.M.P.A. (1996).

[2] Birbrair (L.) and Goldshtein (V.) .- An Example of Noncoincidence of $L_{p-}$ cohomology and Intersection Homology for Real Algebraic varieties. I.M.R.N. 6 (1994), pp. 265-271. 